\title{
The findings of Antimicrobial Resistance Surveillance in Bangladesh (2016-2020)
}

Authors:

Zakir Hossain Habib ${ }^{1}$, Saima binte Golam Rasul ${ }^{1}$, Md. Ashraful Alam ${ }^{2}$, Nazmun Nahar Bably ${ }^{3}$, Iqbal Ansary Khan ${ }^{1}$, S.M. Shahriar Rizvi ${ }^{5 *}$, Tahmina Shirin ${ }^{1}$, Ahmed Nawsher Alam ${ }^{1}$, M. Salim Uzzaman $^{1}$, ASM Alamgir ${ }^{1}$, Mahbubur Rahman ${ }^{1}$, Ashek Ahmmed Shahid Reza ${ }^{1}$, Kazi Mohammad Hassan Ameen ${ }^{6}$, AKM Muraduzzaman ${ }^{7}$, Ishrat Siddiqui ${ }^{1}$, Zarin Tasnim Haider ${ }^{4}$, Meerjady Sabrina $^{2}$ Flora $^{8}$

1 Institute of Epidemiology, Disease Control and Research (IEDCR), Dhaka, Bangladesh.

2 North Bengal Medical College, Sirajganj, Bangladesh.

3 United Nations Children's Fund, Bangladesh.

4 Augmedix BD

5 Communicable Disease Control, Directorate General of Health Services (DGHS), Bangladesh.

6 World Health Organization, Bangladesh.

7 Monash University, Australia.

8 Directorate General of Health Services (DGHS), Dhaka, Bangladesh. 
medRxiv preprint doi: https://doi.org/10.1101/2021.06.12.21251710; this version posted August 10, 2021. The copyright holder for this preprint (which was not certified by peer review) is the author/funder, who has granted medRxiv a license to display the preprint in perpetuity.

It is made available under a CC-BY 4.0 International license .

\section{Abstract}

Surveillance is one of the effective tools to address antimicrobial resistance. In Bangladesh a countrywide antimicrobial resistance surveillance has been ongoing since 2016. The main objective of this surveillance is to formulate the guideline for clinicians and to assist policy makers to know the gravity of the AMR problem in Bangladesh.

It is a case-based surveillance conducted by Institute of Epidemiology, Disease Control \& Research (IEDCR) in nine sentinel sites where five types of clinical cases were selected according to case definition, and ten types of bacteria were identified from six types of preselected specimens. All the laboratory works were performed following the standard operating procedure supplied by the AMR surveillance Reference laboratory at IEDCR. Total 19,263 samples were processed during the period of March 2017 to March 2020 among which wound swab yielded highest growth (57\%). E. coli was the highest (1717) isolated organism among the ten priority pathogens which showed highest sensitivity (91\%) to Imipenem. Imipenem also showed higher sensitivity to most of the organisms. Third generation cephalosporin was found to be less sensitive to Escherichia coli $(37 \%)$ and Klebsiella pneumoniae (28\%); nevertheless, Salmonella species showed higher sensitivity (97\%) to it. Acinetobacter calcoaceticus-baumannii complex isolated from ICU patients showed alarming resistance to all of the antibiotics including highest sensitive antibiotic Imipenem (29\%). Salmonella species isolated from blood showed higher susceptibility to most of the antibiotics except ciprofloxacin (7\%). Alarmingly, only 36\% of the Staphylococcus aureus isolates showed susceptibility to cefoxitin indicates high prevalence of MRSA infection.

The result of the surveillance representing the whole country is surely alarming as many of the bacteria are resistant to the commonly used as well as reserve groups of antibiotics. Concerted effort should be taken from all concerned authorities to curb the problem immediately. 


\section{Introduction}

Antimicrobial resistance (AMR) is one of the most complex and multifaceted health challenges facing the global community. It is regarded as the single biggest threat facing the world in the area of infectious diseases. Excessive and inappropriate antibiotic usage is regarded as the main cause of the emergence of resistant organisms. Drug-resistant infections have already contributed to at least 700,000 deaths a year [1]. In Brazil, Indonesia and Russia, 40 to $60 \%$ of infections are already caused by drug-resistant bacteria, compared to an average of $17 \%$ in Organisation for Economic Co-operation and Development (OECD) countries [2]. Given the current trajectory, drug resistance could lead to 10 million deaths annually and plunge 24 million people into extreme poverty by 2050 [1]. Left unchecked, AMR is likely to become one of the world's largest health threats, surpassing many other major conditions, such as diabetes and cancer; in scale have a severe effect on economies around the world.

Bangladesh, a developing country of Southeast Asia with a high degree of AMR, poses a regional and global threat. In a study performed in Chittagong in 2003, typhoid patients were found to be unresponsive to second-line therapy (ciprofloxacin). First-line therapy was not even attempted because of existing resistance [3]. Therapeutic failures like this are not rare at all. Furthermore, concerning this, multiple studies have demonstrated irrational antibiotic prescribing by physicians, a habit of self-medication among patients, and the indiscriminate use of antibiotics in agriculture and farming in different parts of the country [4]. Even though many studies have been performed on the prevalence of AMR in Bangladesh, no attempts have yet been made to systematically unify them. 
medRxiv preprint doi: https://doi.org/10.1101/2021.06.12.21251710; this version posted August 10, 2021. The copyright holder for this preprint (which was not certified by peer review) is the author/funder, who has granted medRxiv a license to display the preprint in perpetuity.

It is made available under a CC-BY 4.0 International license .

The World Health Organization (WHO) acknowledged AMR as a global public health problem in 1998 and urged member states to take measures to encourage appropriate use of antimicrobials. In May 2015, the World Health Assembly adopted the Global Action Plan on AMR. All countries are required to develop their national action plan based on the GAP. One of the five strategic objectives of the Global Plan is to strengthen the evidence base through surveillance and research. The World Health Organization (WHO) has developed the Global Antimicrobial Resistance Surveillance System (GLASS) to support the implementation of the Global Action Plan on antimicrobial resistance (AMR). GLASS promotes and supports standardized antimicrobial resistance (AMR) surveillance worldwide [1].

In concordance with the global and WHO activities on Antimicrobial Resistance Containment (ARC), the Ministry of Health and Family Welfare (MoHFW) in Bangladesh has come forward and the initiative was taken to conduct the program for containment of antimicrobial resistance in Bangladesh. A National Strategy for ARC in Bangladesh as well as National Action Plan 20172022 was developed. The establishment of a Surveillance System for AMR is emphasized in (NAP). A nationwide AMR Surveillance in human health is being conducted by the Institute of Epidemiology, Disease Control \& Research (IEDCR) since 2016 keeping in line with GLASS as well as country perspective in nine sentinel sites in different geographical locations all over the country to know the status of Antimicrobial resistance pattern of different bacteria.

\section{Materials and Methods}

The AMR surveillance in Bangladesh is a 'case-based surveillance', one of the three surveillance methods designed under the GLASS protocol. With the technical support from the US-CDC through Global Health Security Agenda (GHSA), World Health Organization (WHO) 
medRxiv preprint doi: https://doi.org/10.1101/2021.06.12.21251710; this version posted August 10, 2021. The copyright holder for this preprint (which was not certified by peer review) is the author/funder, who has granted medRxiv a license to display the preprint in perpetuity.

It is made available under a CC-BY 4.0 International license .

and Government of Bangladesh, IEDCR conducted the surveillance for the period of March 2016 to March 2020.

Figure 1: Surveillance activities started in 9 sites all over Bangladesh in 2 phases:

Nine Surveillance Sites of Bangladesh
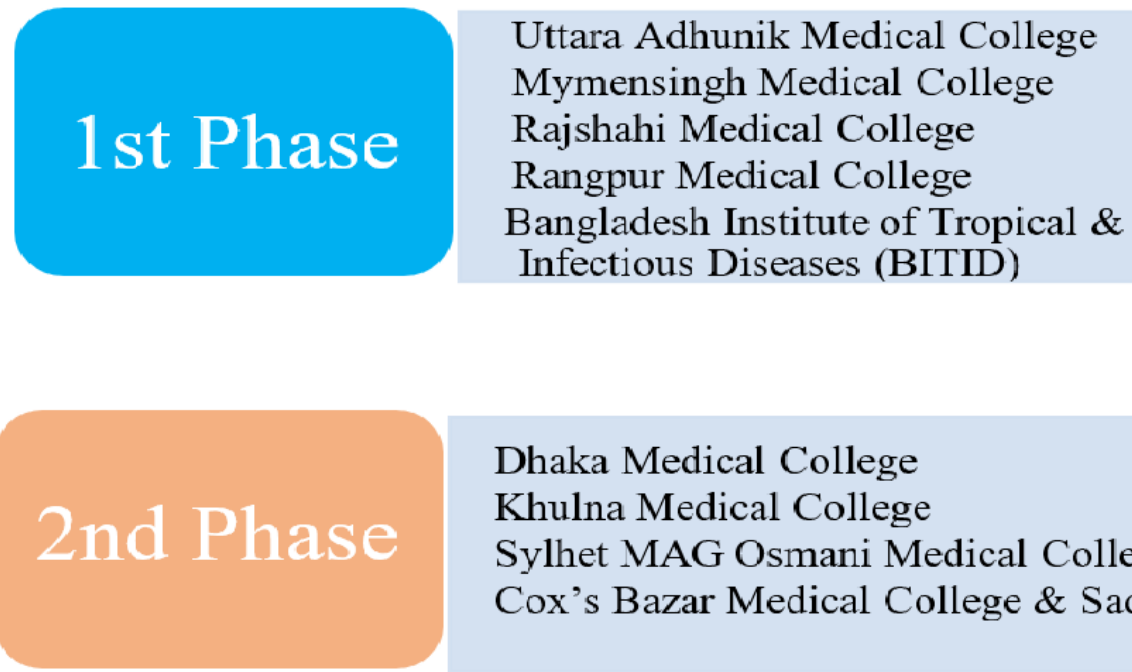

Dhaka Medical College

Khulna Medical College

Sylhet MAG Osmani Medical College

Cox's Bazar Medical College \& Sadar Hospital

Surveillance sites were selected on the basis of geographical representation (Figure 2), ability of the hospital to enroll cases and availability of a microbiology laboratory with capacity to perform bacterial culture and sensitivity tests. Capacity building of the sites included hands-on training (both basic \& refresher), providing of laboratory standard operating procedures (SOPs), providing of instruments, logistics and technical support through the laboratory networking system. 


\section{Figure 2: Geographical Distribution of Surveillance Sites of Bangladesh}

\section{Geographical Distribution of Surveillance Sites of Bangladesh}

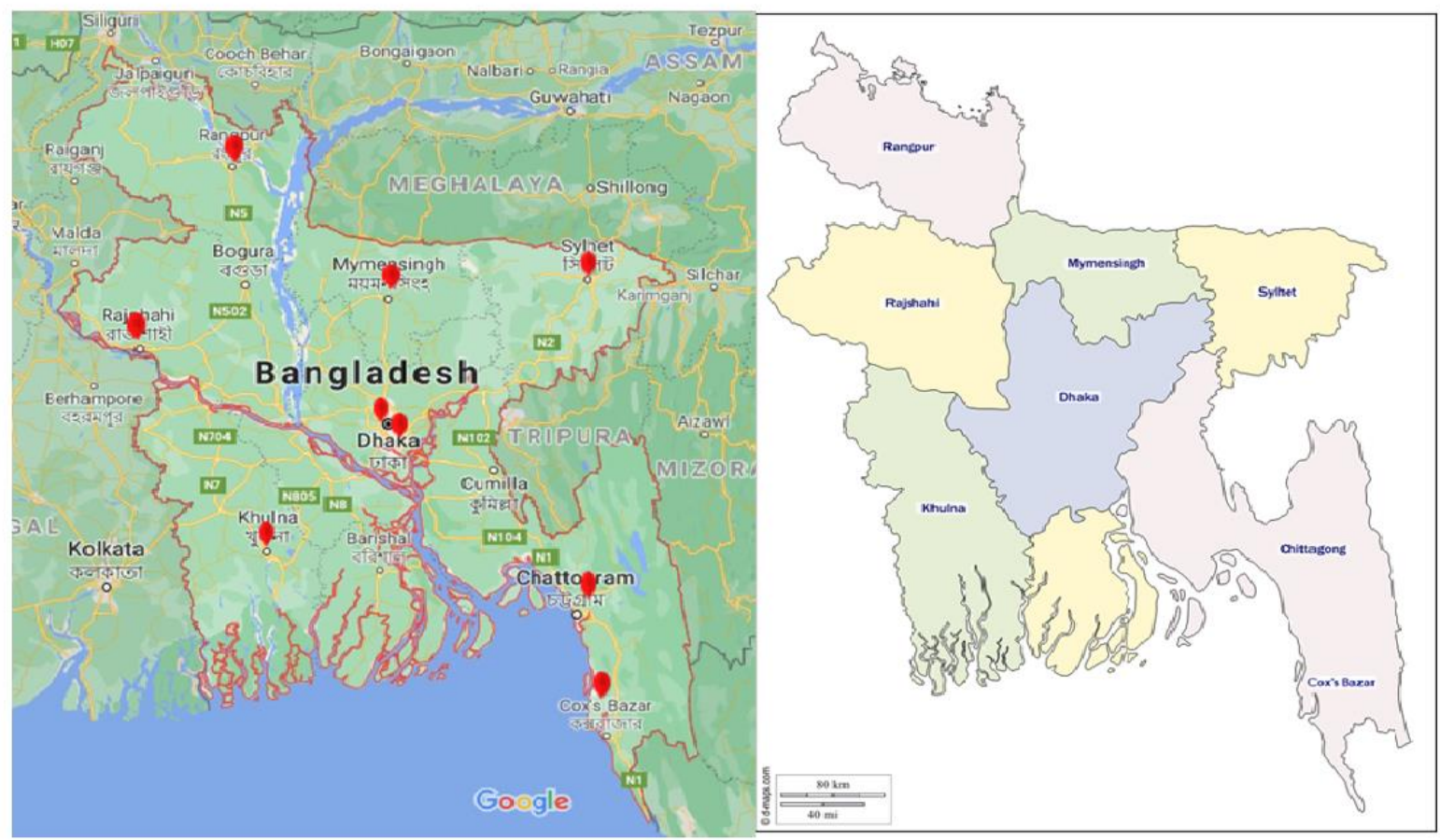

Surveillance activities included collection and testing of samples and compiling of the epidemiological and laboratory data. All the laboratory tests including the Antimicrobial Susceptibility Testing (AST) was done at the sentinel sites. Laboratory works have been performed by strictly following the SOPs provided by the AMR Reference Laboratory at IEDCR. All the relevant epidemiological as well as laboratory data were compiled in a software and the hard copy was maintained. The data were cleansed and analyzed using WHONET software at IEDCR.

Internal and external quality control were ensured. For external quality control, 5\% of the randomly selected isolates of positive samples had been retested at the reference laboratory as well as discordant results were checked at another quality laboratory. The reference laboratory as well as the sentinel sites participated in Proficiency Testing (PT) by the College of American Pathologists (CAP). 
medRxiv preprint doi: https://doi.org/10.1101/2021.06.12.21251710; this version posted August 10, 2021. The copyright holder for this preprint (which was not certified by peer review) is the author/funder, who has granted medRxiv a license to display the preprint in perpetuity.

It is made available under a CC-BY 4.0 International license .

Five different cases of infectious conditions including Urinary tract infection (UTI), diarrhoeal diseases, Wound infection, pneumonia and septicaemia were enrolled from the hospitals by the surveillance physicians following case definitions and specific samples were collected from them according to the protocol which included urine, stool, wound swab, blood, sputum and endotracheal aspirate. In the laboratories, 10 pathogens were identified by biochemical methods from the samples following laboratory SOP and their susceptibility test was done by Kirby-Bauer disc diffusion method following CLSI and also, the zone diameter was noted. The organisms identified were-Escherichia coli, Klebsiella pneumoniae, Enterococcus species (spp)., Vibrio cholerae, Shigella spp. Streptococcus pneumoniae, Staphylococcus aureus, Salmonella spp., Pseudomonas aeruginosa and Acinetobacter calcoaceticus-baumannii (Acb) complex. These 10 pathogens were called the priority pathogens.

\section{Ethical Issue}

Patients were selected according to protocol and before taking sample and epi-data informed written as well as verbal consent were taken and other ethical issues were strictly taken into consideration. The protocol was approved by the lnstitutional Review Board (IRB) of lnstitute of Epidemiology Disease Control and Research (IEDCR). 


\section{Result}

Fig 3: Age \& sex distribution according to specimen type

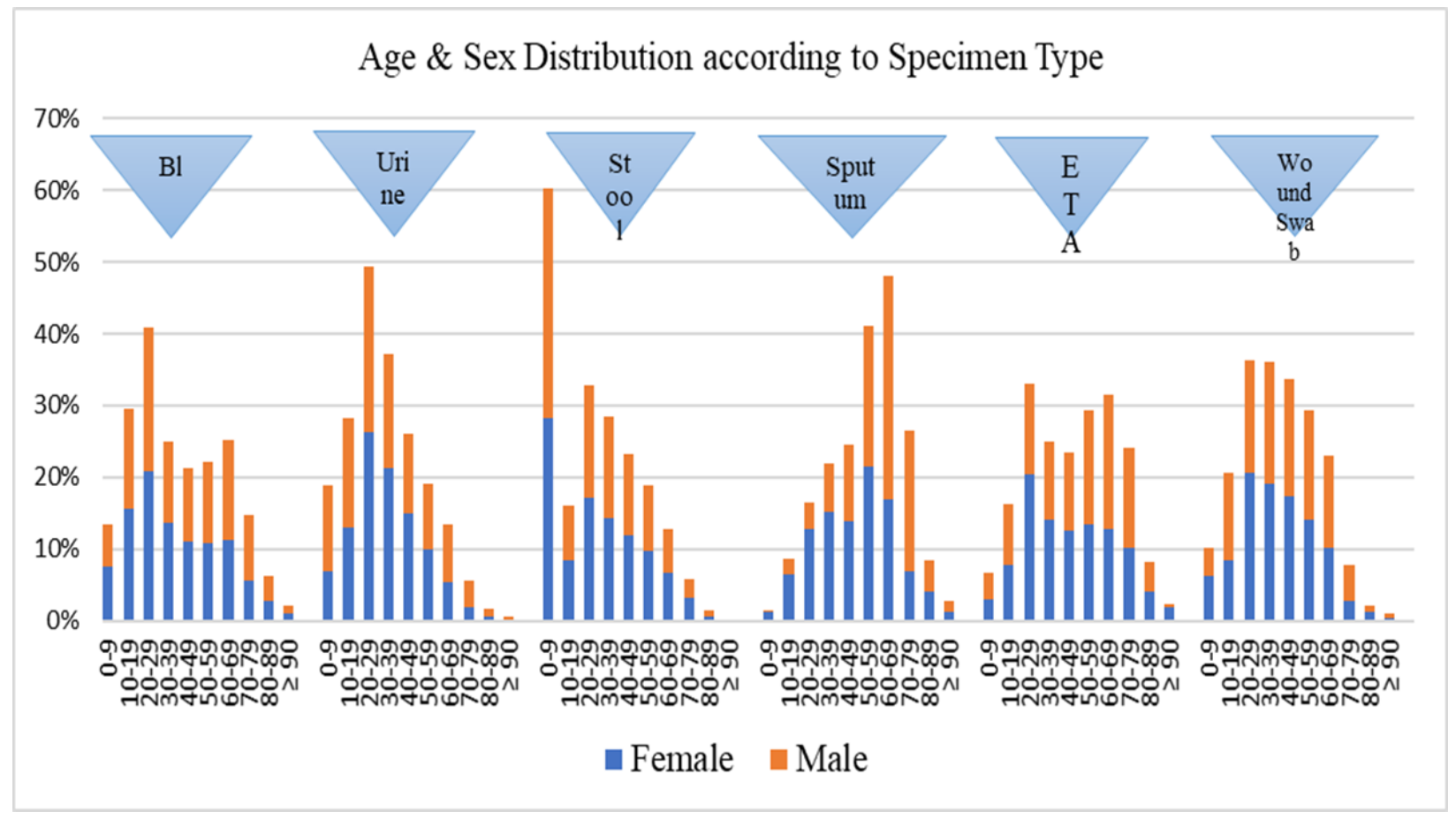

Figure 3 demonstrates the age $\&$ sex distribution according to specimen type.

Except stool and sputum, the other 4 samples were mostly collected from the patients between the age group of 10-29 years. The highest number of stool samples were collected from the age group 0-9 years and the highest number of sputum samples were collected from the age group 60-69 years. As per the sex distribution, there was no significance difference between male and female patients. 
Figure 4 demonstrates distribution of growth in cultured specimen

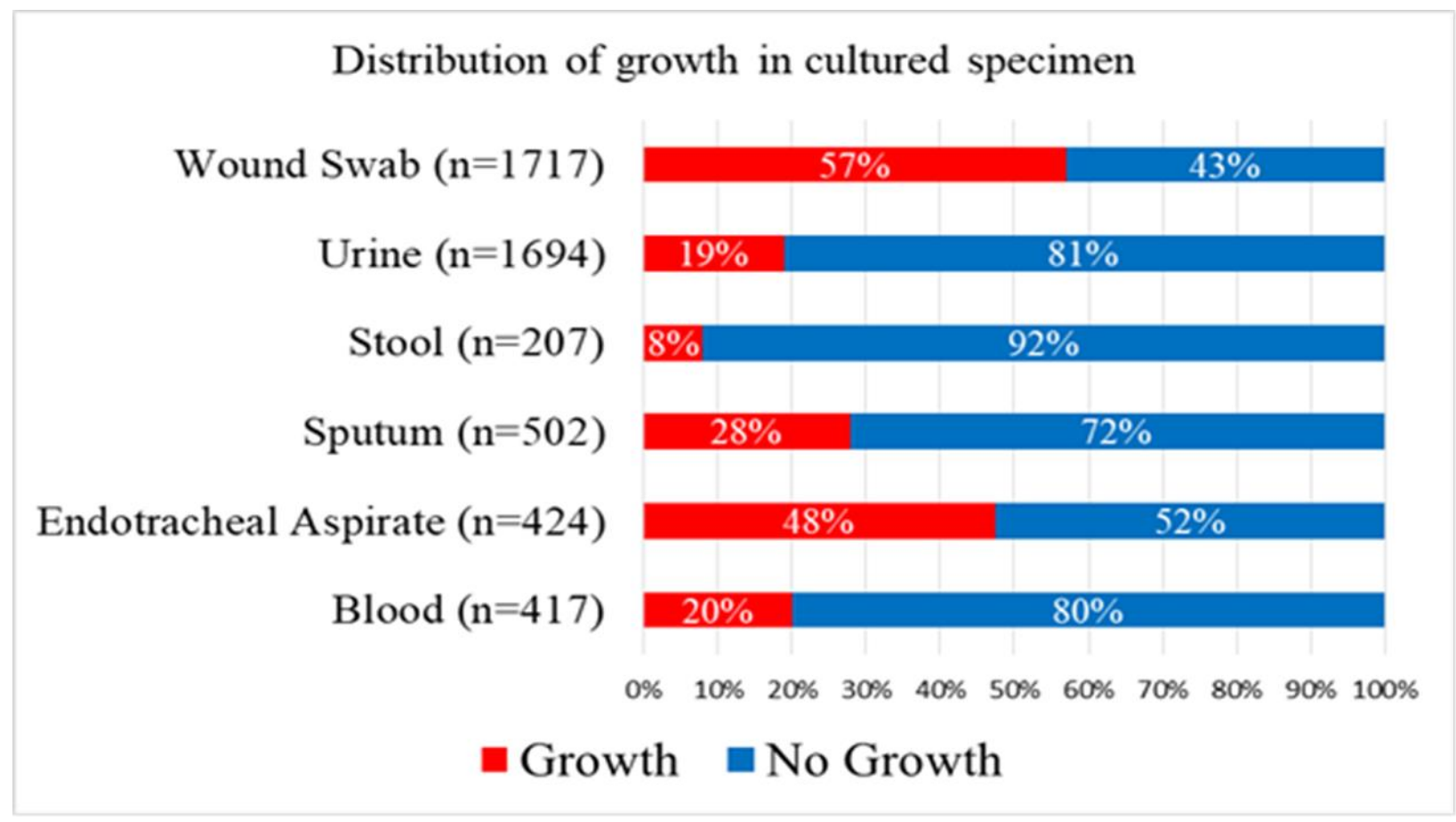

Figure 4 demonstrates distribution of growth in cultured specimen

Wound swab yielded the highest growth among the 6 different samples (57\%) followed by endotracheal aspirate (48\%). On the other hand, stool samples yielded the lowest growth (8\%). Here, the growth of $E$. coli in stool samples was excluded as the pathogenicity test for the isolates was not done.

Table 1: Distribution of priority pathogens according to specimens

\begin{tabular}{|c|c|c|c|c|c|c|}
\hline \multirow{2}{*}{$\begin{array}{l}\text { Priority } \\
\text { Pathogens }\end{array}$} & \multicolumn{6}{|c|}{ Specimens } \\
\hline & $\begin{array}{l}\text { Wound } \\
\text { Swab } \\
(n=1717)\end{array}$ & $\begin{array}{l}\text { Urine } \\
(n=1694)\end{array}$ & $\begin{array}{l}\text { Stool } \\
(n=207)\end{array}$ & $\begin{array}{l}\text { Sputum } \\
(n=502)\end{array}$ & $\begin{array}{l}\text { Endotrachea } \\
\text { l Aspirate } \\
(n=424)\end{array}$ & $\begin{array}{l}\text { Blood } \\
(n=417)\end{array}$ \\
\hline E. coli & $233(14 \%)$ & $\begin{array}{l}1015(60 \% \\
)\end{array}$ & - & $51(10 \%)$ & $35(8 \%)$ & $65(16 \%)$ \\
\hline
\end{tabular}


medRxiv preprint doi: https://doi.org/10.1101/2021.06.12.21251710; this version posted August 10, 2021. The copyright holder for this preprint (which was not certified by peer review) is the author/funder, who has granted medRxiv a license to display the preprint in perpetuity.

It is made available under a CC-BY 4.0 International license .

\begin{tabular}{|l|l|l|l|l|l|l|}
\hline $\begin{array}{l}\text { Klebsiella } \\
\text { pneumoniae }\end{array}$ & $156(9 \%)$ & $278(16 \%)$ & - & $\begin{array}{l}231(46 \% \\
)\end{array}$ & $149(35 \%)$ & $31(7 \%)$ \\
\hline $\begin{array}{l}\text { Enterococcus } \\
\text { species }\end{array}$ & $13(0.76 \%$ & $85(5 \%)$ & - & $2(0.4 \%)$ & - & $1(0.24 \%)$ \\
\hline $\begin{array}{l}\text { Vibrio } \\
\text { cholerae }\end{array}$ & - & - & $110(53 \%$ & - & - & - \\
\hline $\begin{array}{l}\text { Shigella } \\
\text { species }\end{array}$ & - & - & $47(23 \%)$ & - & - & - \\
\hline $\begin{array}{l}\text { Streptococcus } \\
\text { pneumoniae }\end{array}$ & - & - & - & $2(0.4 \%)$ & $1(0.2 \%)$ & $3(0.7 \%)$ \\
\hline $\begin{array}{l}\text { Staphylococcu } \\
\text { s aureus }\end{array}$ & $235(14 \%)$ & $115(7 \%)$ & $2(1 \%)$ & $95(19 \%)$ & $26(6 \%)$ & $36(9 \%)$ \\
\hline $\begin{array}{l}\text { Salmonella } \\
\text { species }\end{array}$ & $1(0.06 \%)$ & - & $45(22 \%)$ & - & - & $243(58 \%$ \\
\hline $\begin{array}{l}\text { Pseudomonas } \\
\text { aeruginosa }\end{array}$ & $779(45 \%)$ & $93(6 \%)$ & - & $51(4 \%)$ & $80(19 \%)$ & $20(5 \%)$ \\
\hline $\begin{array}{l}\text { Acb Complex } \\
\text { Others }\end{array}$ & $52(3 \%)$ & $23(1 \%)$ & $1(0.48 \%)$ & $10(2 \%)$ & $108(26 \%)$ & $12(3 \%)$ \\
\hline $\begin{array}{l}\text { Acb, Acinetobacter } \\
\text { (2a) }\end{array}$ & $85(5 \%)$ & $2(1 \%)$ & $60(12 \%)$ & $25(6 \%)$ & $6(1 \%)$ \\
\hline
\end{tabular}

Acb, Acinetobacter calcoaceticus-baumannii.

Table 1 demonstrates Distribution of priority pathogens according to specimens

Among the 10 priority pathogens, E. coli showed the highest growth (60\%) in the urine samples.

Among the wound swabs, Pseudomonas aeruginosa was the highest isolated organism (45\%) followed by Staph. aureus (14\%) and E. coli (14\%). Vibrio cholera was the most abundant in stool samples (53\%). In the sputum and endotracheal aspirates, Klebsiella pneumoniae showed the highest growth (46\% and $35 \%$ respectively). Acb complex was the second highest organism identified from the endotracheal aspirate (26\%). In blood, Salmonella spp. were the highest isolated $(58 \%)$ pathogens. 
medRxiv preprint doi: https://doi.org/10.1101/2021.06.12.21251710; this version posted August 10, 2021. The copyright holder for this preprint (which was not certified by peer review) is the author/funder, who has granted medRxiv a license to display the preprint in perpetuity.

It is made available under a CC-BY 4.0 International license .

Table 2: Antibiotic susceptibility of priority pathogens irrespective of specimens

\begin{tabular}{|c|c|c|c|c|c|c|c|c|c|c|}
\hline & \multicolumn{10}{|c|}{ Priority Pathogens } \\
\hline $\begin{array}{c}\text { Antibiotic } \\
\text { Name }\end{array}$ & 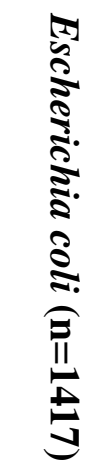 & 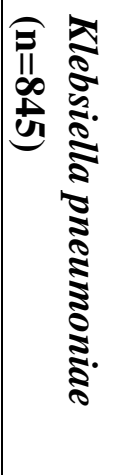 & 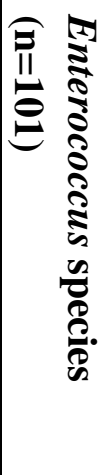 & 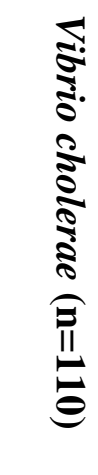 & 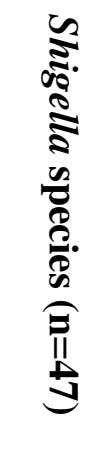 & 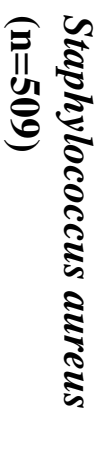 & 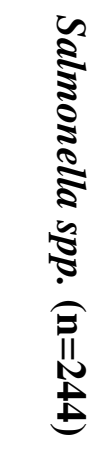 & 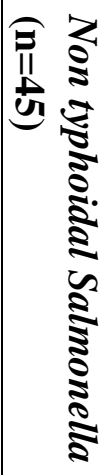 & 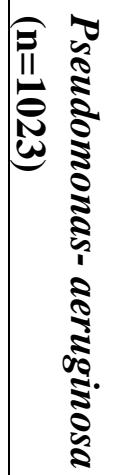 & 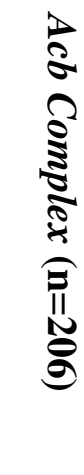 \\
\hline Amikacin & $80 \%$ & $63 \%$ & - & - & - & - & $98 \%$ & - & $35 \%$ & $27 \%$ \\
\hline $\begin{array}{l}\text { Amoxicillin- } \\
\text { Clavulanate }\end{array}$ & $26 \%$ & $16 \%$ & - & - & - & - & $85 \%$ & - & - & - \\
\hline Ampicillin & $10 \%$ & $3 \%$ & $60 \%$ & - & $36 \%$ & - & $74 \%$ & $26 \%$ & - & - \\
\hline Aztreonam & $34 \%$ & $29 \%$ & - & - & - & - & $94 \%$ & - & $20 \%$ & - \\
\hline Azithromycin & - & - & - & $83 \%$ & $53 \%$ & $12 \%$ & - & $*$ & - & - \\
\hline Ceftazidime & $40 \%$ & $29 \%$ & - & - & - & - & $98 \%$ & - & $20 \%$ & $7 \%$ \\
\hline Clindamycin & - & - & - & - & - & $41 \%$ & - & - & - & - \\
\hline Ciprofloxacin & $39 \%$ & $37 \%$ & $38 \%$ & $84 \%$ & $25 \%$ & $30 \%$ & $7 \%$ & $49 \%$ & $23 \%$ & $12 \%$ \\
\hline Cephalexin & $26 \%$ & $20 \%$ & - & - & - & - & $94 \%$ & - & - & - \\
\hline Cefepime & $46 \%$ & $39 \%$ & - & - & - & - & $98 \%$ & - & $26 \%$ & $13 \%$ \\
\hline Ceftriaxone & $37 \%$ & $28 \%$ & - & - & $89 \%$ & - & $97 \%$ & $70 \%$ & - & $9 \%$ \\
\hline Cefoxitin & - & - & - & - & - & $36 \%$ & - & - & - & - \\
\hline Cefuroxime & $30 \%$ & $25 \%$ & - & - & - & - & $95 \%$ & - & - & - \\
\hline Doxycycline & - & - & - & - & - & $66 \%$ & - & - & - & $27 \%$ \\
\hline Erythromycin & - & - & - & $7 \%$ & - & - & - & - & - & - \\
\hline Gentamicin & $70 \%$ & $57 \%$ & $40 \%$ & - & - & $63 \%$ & $98 \%$ & - & $25 \%$ & $20 \%$ \\
\hline Imipenem & $91 \%$ & $77 \%$ & - & - & - & - & $100 \%$ & - & $53 \%$ & $29 \%$ \\
\hline Linezolid & - & - & $76 \%$ & - & - & - & - & - & - & - \\
\hline
\end{tabular}


medRxiv preprint doi: https://doi.org/10.1101/2021.06.12.21251710; this version posted August 10, 2021. The copyright holder for this preprint (which was not certified by peer review) is the author/funder, who has granted medRxiv a license to display the preprint in perpetuity.

It is made available under a CC-BY 4.0 International license .

\begin{tabular}{|l|c|c|c|c|c|c|c|c|c|c|}
\hline Mecillinam & - & - & - & - & $64 \%$ & - & - & - & - & - \\
\hline Nitrofurantoin & $77 \%$ & $46 \%$ & $75 \%$ & - & - & - & - & - & - & - \\
\hline Norfloxacin & $45 \%$ & $49 \%$ & - & - & - & - & $79 \%$ & - & $18 \%$ & - \\
\hline Oxacillin & - & - & - & - & - & $21 \%$ & - & - & - & - \\
\hline Piperacillin & $22 \%$ & $17 \%$ & - & - & - & - & $74 \%$ & - & $30 \%$ & $6 \%$ \\
\hline $\begin{array}{l}\text { Piperacillin- } \\
\text { Tazobactam }\end{array}$ & - & - & - & - & - & - & - & - & $38 \%$ & $20 \%$ \\
\hline Penicillin-G & - & - & $38 \%$ & - & - & $11 \%$ & - & - & - & - \\
\hline Rifampicin & - & - & - & - & - & $69 \%$ & - & - & - & - \\
\hline $\begin{array}{l}\text { Sulfamethoxazol } \\
\text { e-Trimethoprim }\end{array}$ & $41 \%$ & $31 \%$ & - & $4 \%$ & $37 \%$ & $49 \%$ & $82 \%$ & $51 \%$ & - & $21 \%$ \\
\hline Tetracycline & $42 \%$ & $37 \%$ & $26 \%$ & $57 \%$ & - & - & $85 \%$ & - & - & - \\
\hline
\end{tabular}

*Less than 30 isolates tested.

Table 2 demonstrates the antibiotic susceptibility of priority pathogens irrespective of specimen.

A varied range of antibiotic susceptibility is found in different bacteria. Most of the bacteria were susceptible to Imipenem; among them Salmonella was $100 \%$ susceptible. Ampicillin was less effective against pathogens like E. coli, Klebsiella pneumoniae and Non-typhoidal Salmonella. Salmonella spp. showed high susceptibility to almost all antibiotics. Among the 10 priority pathogens, Pseudomonas aeruginosa and Acb complex showed low sensitivity to almost all the antibiotics. Pseudomonas aeruginosa showed less than $40 \%$ sensitivity to all the antibiotics except imipenem and Acb complex showed less than $30 \%$ sensitivity to all of the antibiotics. Only $36 \%$ Staphylococcus aureus isolates were susceptible to Cefoxitin whereas Clindamycin was sensitive to $41 \%$ isolates.

The susceptibility patterns of Staphylococcus aureus to vancomycin and Linezolid are not mentioned here as those could not be confirmed by MIC testing. 
Table: 3 Antibiogram of Urine

\begin{tabular}{|c|c|c|c|c|c|}
\hline $\begin{array}{l}\text { Antibiotic } \\
\text { Name }\end{array}$ & $\begin{array}{l}\text { Escherichia } \\
\text { coli } \\
(\mathrm{n}=1015)\end{array}$ & $\begin{array}{l}\text { Klebsiella } \\
\text { pneumoniae } \\
(\mathrm{n}=278)\end{array}$ & $\begin{array}{l}\text { Enterococcu } \\
s \quad \text { species } \\
(\mathrm{n}=85)\end{array}$ & $\begin{array}{l}\text { Staphylococ } \\
\text { cus aureus } \\
(\mathrm{n}=115)\end{array}$ & $\begin{array}{l}\text { Pseudomon } \\
\text { as } \\
\text { aeruginosa } \\
(\mathrm{n}=93)\end{array}$ \\
\hline Amikacin & $86 \%$ & $82 \%$ & - & - & $69 \%$ \\
\hline $\begin{array}{l}\text { Amoxicillin- } \\
\text { Clavulanate }\end{array}$ & $30 \%$ & $31 \%$ & - & - & - \\
\hline Ampicillin & $12 \%$ & $5 \%$ & $61 \%$ & - & - \\
\hline Aztreonam & $39 \%$ & $51 \%$ & - & - & $30 \%$ \\
\hline Azithromycin & - & - & - & $7 \%$ & - \\
\hline Ceftazidime & $45 \%$ & $54 \%$ & - & - & $36 \%$ \\
\hline Clindamycin & - & - & - & $45 \%$ & - \\
\hline Ciprofloxacin & $44 \%$ & $57 \%$ & $41 \%$ & $41 \%$ & $40 \%$ \\
\hline Cephalexin & $29 \%$ & $36 \%$ & - & - & - \\
\hline Cefepime & $52 \%$ & $63 \%$ & - & - & $44 \%$ \\
\hline Ceftriaxone & $45 \%$ & $55 \%$ & - & - & - \\
\hline Cefoxitin & - & - & - & $20 \%$ & - \\
\hline Cefuroxime & $35 \%$ & $43 \%$ & - & - & - \\
\hline Doxycycline & - & - & - & $70 \%$ & - \\
\hline Gentamicin & $78 \%$ & $75 \%$ & $46 \%$ & $75 \%$ & $63 \%$ \\
\hline Imipenem & $95 \%$ & $92 \%$ & - & - & $74 \%$ \\
\hline Linezolid & - & - & $80 \%$ & - & - \\
\hline Nitrofurantoin & $80 \%$ & $51 \%$ & $75 \%$ & - & - \\
\hline Norfloxacin & $46 \%$ & $59 \%$ & - & - & $*$ \\
\hline Oxacillin & - & - & - & $7 \%$ & - \\
\hline Piperacillin & $25 \%$ & $32 \%$ & - & - & $53 \%$ \\
\hline
\end{tabular}




\begin{tabular}{|l|c|c|c|c|c|}
\hline $\begin{array}{l}\text { Piperacillin- } \\
\text { Tazobactam }\end{array}$ & - & - & - & - & $73 \%$ \\
\hline Penicillin-G & - & - & $37 \%$ & $24 \%$ & - \\
\hline Rifampicin & - & - & - & $83 \%$ & - \\
\hline $\begin{array}{l}\text { Sulfamethoxazole } \\
\text {-Trimethoprim }\end{array}$ & $45 \%$ & $45 \%$ & - & $53 \%$ & - \\
\hline Tetracycline & $45 \%$ & $49 \%$ & $26 \%$ & - & - \\
\hline
\end{tabular}

*Less than 30 isolates tested.

Table 3 demonstrates antibiogram of urine sample

Five types of pathogens were identified from the urine sample, where E. coli was highest, followed by Klebsiella pneumoniae. Imipenem was the most effective against the pathogens identified from the urine samples. More than $90 \%$ of isolates of E. coli and Klebsiella pneumoniae were sensitive to Imipenem whereas ampicillin showed lowest sensitivity to both of these organisms (12\% and $5 \%$ respectively). Only $45 \%$ of the $E$. coli were susceptible to Ceftriaxone. Nitrofurantoin was sensitive to $80 \%$ of $E$. coli. The antibiotics showed relatively less sensitivity to $S$. aureus and $P$. aeruginosa pathogens. About $20 \%$ and $45 \%$ of the S. aureus isolates were sensitive to Cefoxitin and clindamycin respectively.

Table: 4 Antibiogram of Wound Swab

\begin{tabular}{|l|l|l|l|l|}
\hline Antibiotic & $\begin{array}{l}\text { Escherichia } \\
\text { coli }(\mathbf{n = 2 3 3})\end{array}$ & $\begin{array}{l}\text { Klebsiella } \\
\text { pneumoniae } \\
(\mathbf{n = 1 5 6})\end{array}$ & $\begin{array}{l}\text { Staphylococcus } \\
\text { aureus }(\mathbf{n = 2 3 5})\end{array}$ & $\begin{array}{l}\text { Pseudomonas } \\
\text { aeruginosa }(\mathbf{n}=779)\end{array}$ \\
\hline Amikacin & $59 \%$ & $40 \%$ & - & $29 \%$ \\
\hline $\begin{array}{l}\text { Amoxicillin- } \\
\text { Clavulanate }\end{array}$ & $8 \%$ & $6 \%$ & - & - \\
\hline Ampicillin & $2 \%$ & $0 \%$ & - & - \\
\hline Aztreonam & $14 \%$ & $12 \%$ & - & $18 \%$ \\
\hline Azithromycin & - & - & $16 \%$ & - \\
\hline
\end{tabular}




\begin{tabular}{|c|c|c|c|c|}
\hline Ceftazidime & $26 \%$ & $9 \%$ & - & $16 \%$ \\
\hline Clindamycin & - & - & $47 \%$ & - \\
\hline Ciprofloxacin & $24 \%$ & $17 \%$ & $25 \%$ & $20 \%$ \\
\hline Cephalexin & $10 \%$ & $7 \%$ & - & - \\
\hline Cefepime & $29 \%$ & $18 \%$ & - & $23 \%$ \\
\hline Ceftriaxone & $14 \%$ & $9 \%$ & - & - \\
\hline Cefoxitin & - & - & $50 \%$ & - \\
\hline Cefuroxime & $11 \%$ & $8 \%$ & - & - \\
\hline Doxycycline & - & - & $66 \%$ & - \\
\hline Gentamicin & $45 \%$ & $34 \%$ & $65 \%$ & $19 \%$ \\
\hline Imipenem & $75 \%$ & $73 \%$ & - & $51 \%$ \\
\hline Linezolid & - & - & - & - \\
\hline Norfloxacin & $32 \%$ & $*$ & - & $15 \%$ \\
\hline Oxacillin & - & - & $25 \%$ & - \\
\hline Piperacillin & $11 \%$ & $10 \%$ & - & $28 \%$ \\
\hline $\begin{array}{l}\text { Piperacillin- } \\
\text { Tazobactam }\end{array}$ & - & - & - & $33 \%$ \\
\hline Penicillin-G & - & - & $6 \%$ & - \\
\hline Rifampicin & - & - & $76 \%$ & - \\
\hline $\begin{array}{l}\text { Sulfamethoxaz } \\
\text { ole- } \\
\text { Trimethoprim }\end{array}$ & $27 \%$ & $17 \%$ & $54 \%$ & - \\
\hline Tetracycline & $20 \%$ & $27 \%$ & - & - \\
\hline
\end{tabular}

*Less than 30 isolates tested.

Table 4 demonstrates the antibiogram of the wound swab samples.

Both E. coli and K. pneumoniae showed highest susceptibility (75\% and $73 \%$ respectively) to Imipenem. Ceftriaxone showed sensitivity to only $14 \%$ and $9 \%$ of E. coli and K. pneumoniae isolates respectively. Other than Imipenem, other antibiotics were sensitive to less than $35 \%$ of $P$. 
medRxiv preprint doi: https://doi.org/10.1101/2021.06.12.21251710; this version posted August 10, 2021. The copyright holder for this preprint (which was not certified by peer review) is the author/funder, who has granted medRxiv a license to display the preprint in perpetuity.

It is made available under a CC-BY 4.0 International license.

aeruginosa. Most S. aureus (76\%) were susceptible to Rifampicin whereas fewest (6\%) were susceptible to Penicillin- G. Half of the S. aureus isolates were susceptible to cefoxitin and $47 \%$ of the isolates showed susceptibility to clindamycin.

Table: 5 Antibiogram of Stool

\begin{tabular}{|l|l|l|l|}
\hline Antibiotic Name & $\begin{array}{l}\text { Vibrio cholerae } \\
(\mathbf{n = 1 1 0})\end{array}$ & $\begin{array}{l}\text { Shigella species } \\
(\mathbf{n = 4 7})\end{array}$ & $\begin{array}{l}\text { Salmonella } \\
\text { species (n=45) }\end{array}$ \\
\hline Ampicillin & - & $36 \%$ & $26 \%$ \\
\hline Azithromycin & $83 \%$ & $*$ & $*$ \\
\hline Ciprofloxacin & $84 \%$ & $25 \%$ & $49 \%$ \\
\hline Ceftriaxone & - & $89 \%$ & - \\
\hline Erythromycin & $7 \%$ & - & - \\
\hline Mecillinam & - & $64 \%$ & $51 \%$ \\
\hline Sulfamethoxazole-Trimethoprim & $4 \%$ & $37 \%$ & - \\
\hline Tetracycline & $57 \%$ & - & - \\
\hline
\end{tabular}

*Less than 30 isolates tested.

Table 5 demonstrates the antibiogram of the Stool sample.

Ceftriaxone showed the highest sensitivity to Shigella and Salmonella species (89\% and $70 \%$ respectively). Ciprofloxacin showed the highest sensitivity (84\%) to V. cholerae, followed by Azithromycin (83\%), their sensitivity being almost similar.

Table: 6 Antibiogram of Sputum

\begin{tabular}{|l|l|l|}
\hline Antibiotic Name & $\begin{array}{l}\text { Klebsiella } \\
\text { pneumoniae }(\mathbf{n}=\mathbf{2 3 1})\end{array}$ & $\begin{array}{l}\text { Staphylococcus } \\
\text { aureus }(\mathbf{n}=95)\end{array}$ \\
\hline Amikacin & $80 \%$ & - \\
\hline Amoxicillin-Clavulanate & $9 \%$ & - \\
\hline
\end{tabular}




\begin{tabular}{|l|l|l|}
\hline Ampicillin & $2 \%$ & - \\
\hline Aztreonam & $27 \%$ & - \\
\hline Azithromycin & - & $12 \%$ \\
\hline Ceftazidime & $25 \%$ & - \\
\hline Clindamycin & - & $30 \%$ \\
\hline Ciprofloxacin & $43 \%$ & $30 \%$ \\
\hline Cephalexin & $12 \%$ & - \\
\hline Cefepime & $44 \%$ & - \\
\hline Ceftriaxone & $29 \%$ & - \\
\hline Cefuroxime & $21 \%$ & - \\
\hline Doxycycline & - & $64 \%$ \\
\hline Gentamicin & $72 \%$ & $65 \%$ \\
\hline Imipenem & $86 \%$ & - \\
\hline Norfloxacin & $50 \%$ & - \\
\hline Piperacillin & $10 \%$ & - \\
\hline Penicillin-G & $35 \%$ & - \\
\hline Sulfamethoxazole-Trimethoprim & $31 \%$ & - \\
\hline Tetracycline & & - \\
\hline
\end{tabular}

Table 6 demonstrates the antibiogram of the sputum sample.

Imipenem showed the highest sensitivity to K. pneumoniae (86\%), followed by Amikacin (80\%).

Gentamicin showed the highest sensitivity to S. aureus. S. pneumoniae was not included in the table as the number of the isolates was less than 30 . 
Table: 7 Antibiogram of ETA

\begin{tabular}{|c|c|c|c|}
\hline Antibiotic Name & $\begin{array}{l}\text { Klebsiella } \\
\text { pneumoniae } \\
(\mathrm{n}=149)\end{array}$ & $\begin{array}{l}\text { Pseudomonas } \\
\text { aeruginosa }(\mathrm{n}=80)\end{array}$ & $\begin{array}{l}\text { Acb Complex } \\
(\mathrm{n}=108)\end{array}$ \\
\hline Amikacin & $28 \%$ & $25 \%$ & $16 \%$ \\
\hline Amoxicillin-Clavulanate & $5 \%$ & - & - \\
\hline Ampicillin & $0 \%$ & - & - \\
\hline Aztreonam & $3 \%$ & $10 \%$ & - \\
\hline Ceftazidime & $4 \%$ & $5 \%$ & $2 \%$ \\
\hline Ciprofloxacin & $9 \%$ & $12 \%$ & $4 \%$ \\
\hline Cephalexin & $0 \%$ & - & - \\
\hline Cefepime & $7 \%$ & $6 \%$ & $3 \%$ \\
\hline Ceftriaxone & $2 \%$ & - & $4 \%$ \\
\hline Cefuroxime & $1 \%$ & - & - \\
\hline Doxycycline & - & - & $28 \%$ \\
\hline Gentamicin & $24 \%$ & $13 \%$ & $7 \%$ \\
\hline Imipenem & $37 \%$ & $25 \%$ & $15 \%$ \\
\hline Piperacillin & $1 \%$ & $14 \%$ & $3 \%$ \\
\hline Piperacillin-Tazobactam & - & $*$ & $4 \%$ \\
\hline Sulfamethoxazole-Trimethoprim & $12 \%$ & - & $15 \%$ \\
\hline Tetracycline & $14 \%$ & - & - \\
\hline
\end{tabular}

*Less than 30 isolates tested.

Table 7 demonstrates the antibiogram of endotracheal aspirate pathogens

Endotracheal aspirate was taken from the ICU patients and all the antibiotics showed low sensitivity to the identified pathogens from this sample (less than $40 \%$ ). Imipenem was sensitive to only $37 \% \mathrm{~K}$. pneumoniae, which was the highest and only to $25 \%$ of $P$. aeruginosa. Amikacin 
was sensitive to $28 \%$ of $K$. pneumoniae and $25 \%$ of $P$. aeruginosa. Only $20 \%$ or less Acb complex isolates were sensitive to all antibiotics. Cefepime, a Reserve drug, showed sensitivity to less than $10 \%$ of all 3 pathogens.

Table: 8 Antibiogram of Blood

\begin{tabular}{|c|c|c|c|c|}
\hline $\begin{array}{l}\text { Antibiotic } \\
\text { Name }\end{array}$ & $\begin{array}{l}E . \\
(n=65)\end{array} \quad$ coli & $\begin{array}{l}\text { Klebsiella } \\
\text { pneumoniae } \\
(\mathrm{n}=31)\end{array}$ & $\begin{array}{l}\text { Staphylococcus } \\
\text { aureus }(\mathrm{n}=36)\end{array}$ & $\begin{array}{l}\text { Salmonella } \\
\text { species } \\
(\mathrm{n}=243)\end{array}$ \\
\hline Amikacin & $74 \%$ & $50 \%$ & - & $98 \%$ \\
\hline $\begin{array}{l}\text { Amoxicillin- } \\
\text { Clavulanate }\end{array}$ & $27 \%$ & $*$ & - & $85 \%$ \\
\hline Ampicillin & $9 \%$ & $*$ & - & $74 \%$ \\
\hline Aztreonam & $24 \%$ & $*$ & - & $94 \%$ \\
\hline Azithromycin & - & - & $13 \%$ & - \\
\hline Ceftazidime & $30 \%$ & $33 \%$ & - & $98 \%$ \\
\hline Clindamycin & - & - & $35 \%$ & - \\
\hline Ciprofloxacin & $32 \%$ & $27 \%$ & $42 \%$ & $7 \%$ \\
\hline Cephalexin & $17 \%$ & $*$ & $*$ & $94 \%$ \\
\hline Cefepime & $32 \%$ & $*$ & - & $98 \%$ \\
\hline Ceftriaxone & $18 \%$ & $*$ & - & $97 \%$ \\
\hline Cefuroxime & $19 \%$ & $*$ & - & $95 \%$ \\
\hline Doxycycline & - & - & $80 \%$ & - \\
\hline Gentamicin & $63 \%$ & $*$ & $57 \%$ & $98 \%$ \\
\hline Imipenem & $89 \%$ & $*$ & - & $100 \%$ \\
\hline Norfloxacin & $15 \%$ & $*$ & - & $82 \%$ \\
\hline Piperacillin & $14 \%$ & $*$ & - & $74 \%$ \\
\hline $\begin{array}{l}\text { Sulfamethoxaz } \\
\text { ole- } \\
\text { Trimethoprim }\end{array}$ & $25 \%$ & $23 \%$ & $*$ & $82 \%$ \\
\hline
\end{tabular}




\begin{tabular}{|l|l|l|l|l|}
\hline Tetracycline & $46 \%$ & $*$ & - & $86 \%$ \\
\hline
\end{tabular}

*Less than 30 isolates tested.

Table 8 demonstrates the antibiogram of blood samples.

Among the identified pathogens from the blood sample, Salmonella was found to be most abundant. Almost all the antibiotics other than Ciprofloxacin were sensitive to $70 \%$ and above of the all Salmonella species. Ciprofloxacin showed sensitivity to $7 \%$ isolates of the Salmonella only. Imipenem was found to be sensitive to all Salmonella isolates. E. coli was found to be highly susceptible to Imipenem (89\%). Eighty percent of S. aureus were susceptible to Doxycycline. Almost $50 \%$ or more of all identified pathogens were susceptible to Amikacin.

\section{Discussion}

Like other LMICs, Bangladesh is also facing the problem of AMR and the extent of this AMR is not clear due to the lack of adequate data. The AMR surveillance in Bangladesh is the first of its kind which covered all geographical areas in Bangladesh to find out the resistance pattern of ten important bacterial pathogens from six types of samples collected from patients attending outdoors and indoors presented with five clinical syndromes.

Total 19,263 samples were processed. Other than stool and sputum, most of the samples were collected from the age group of 10-29 years. Stool sample was mostly taken from the pediatric department (the age group was between 0 and 9 years). Some of the stool samples were also collected from the Infectious Disease Hospital and ORT unit of Medical college hospitals. Sputum was mostly collected from the age group of 60-69 years. The gender distribution showed no significant variation in terms of collection of samples. 
Even though among the collected samples, urine was the highest in number, it yielded only $19 \%$ growth. A study done in a tertiary hospital in Bangladesh from outdoor and indoor samples yielded similar results where $20.1 \%$ showed significant growth of bacteria (5).

The lowest growth in culture was yielded by stool (9\%). This may be due to exclusion of growth of E. coli as its pathogenicity test could not be done. Wound swab and ETA yielded the highest growth, the percentage being $57 \%$ and $48 \%$ respectively.

Among the gram negative pathogens, E. coli showed the highest (28\%) growth followed by Pseudomonas aeruginosa (21\%), Klebsiella pneumonia (13\%), Salmonella species (6\%), Acb complex (4\%), Vibrio cholera (2\%) and Shigella species (1\%).

E.coli yielded highest growth $(60 \%)$ in urine which is in line with other studies like one conducted in a tertiary care hospital in Bangladesh where E. coli $(58.18 \%)$ was the most prevalent bacteria isolated from positive urine samples (6) and also E. coli showed mostly resistant to penicillins and cephalosporins. Protein synthesis inhibitors also showed poor susceptibility patterns. In UTI, nitrofurantoin is the only oral drug that showed a better (80\%) susceptibility profile. Imipenem was the most effective against $E$. coli followed by Amikacin. E. coli isolated from the wound swab showed a more resistant profile than other samples. In Indian surveillance system, they found colistin, imipenem, meropenem, amikacin and gentamicin as effective drugs which have a concordance with the present study (7). They also found combinations like piperacillin-tazobactam and cefoperazone-sulbactam as effective drugs, which could not be evaluated in our setup. The Indian studies found ESBL producing E. coli were responsible for such resistance patterns for penicillin and cephalosporin $(8,9)$.

Pseudomonas species showed the second highest growth following E. coli. It is the highest isolated pathogen in wound swab (45\%). The samples were collected mainly from the surgery ward 
medRxiv preprint doi: https://doi.org/10.1101/2021.06.12.21251710; this version posted August 10, 2021. The copyright holder for this preprint (which was not certified by peer review) is the author/funder, who has granted medRxiv a license to display the preprint in perpetuity.

It is made available under a CC-BY 4.0 International license .

followed by the burn unit and medicine unit. A study in Nigeria showed similar result where Pseudomonas aeruginosa was the highest (33.3\%) isolated pathogen in post-operative patients followed by Staphylococcus aureus (21.7\%) (10). While a tertiary care hospital in Nepal, pus and wound swab samples from paediatric patients $S$. aureus was the highest isolated organism followed by $P$. aeruginosa (11).

The overall susceptibility pattern of Pseudomonas was very poor. Pseudomonas were highest susceptible (53\%) to Imipenem followed by Piperacillin-Tazobactam (38\%) and Amikacin (35\%). Only Piperacillin showed $38 \%$ sensitivity to Pseudomonas. All other antibiotics showed poor sensitivity to Pseudomonas. Isolates of Pseudomonas aeruginosa from ETA were most resistant. Studies across India showed better susceptibility patterns with colistin followed by aminoglycosides (amikacin/ gentamicin), piperacillin/tazobactam, cephalosporins, fluoroquinolones and carbapenems (imipenem/ meropenem) (7, 12, 13). Multidrug resistance pattern of Pseudomonas aeruginosa has already been established (14).

K. pneumoniae mostly grew from sputum (46\%) and ETA (35\%). A study done in Nigeria also found $K$. pneumoniae as the highest isolated pathogen followed by $H$. influenzae and then $S$. aureus (15). Another study done in a tertiary care hospital, Bali, Indonesia found $K$. pneumoniae as the highest isolated pathogen followed by Acinetobacter baumannii (16).

In this study ETA was cultured from the ventilated patient's samples at ICU and $K$. pneumoniae was found to be the highest pathogen followed by Acb complex. Most of the bacteria isolated were gram negative bacilli (86\%). Among the gram positive bacteria, S. aureus was the highest one (6\%). A prospective study done in a tertiary care hospital in Pondicherry, India showed similar results: most cases of Ventilator Associated Pneumonia (VAP) were caused by Gram negative bacteria, which accounted for $80.9 \%$ of the causative organisms. Acinetobacter baumannii (23.4\%) 
medRxiv preprint doi: https://doi.org/10.1101/2021.06.12.21251710; this version posted August 10, 2021. The copyright holder for this preprint (which was not certified by peer review) is the author/funder, who has granted medRxiv a license to display the preprint in perpetuity.

It is made available under a CC-BY 4.0 International license .

and Pseudomonas aeruginosa (21.3\%) were the predominant Gram-negative bacteria associated with VAP, and Staphylococcus aureus (14.9\%) was the most common Gram-positive bacterium among patients with VAP (17).

The overall susceptibility pattern of $K$. pneumoniae showed below $50 \%$ for most of the antibiotics except Imipenem (77\%), Amikacin (63\%) and gentamicin (57\%). Isolates from ETA showed the most resistant profile than others. Susceptibility showed diversity in India with high rates of ESBL production. Susceptibility to carbapenems showed a wide range from 44 to $72 \%$, amikacin showed $65 \%$ while gentamicin susceptibility was $55 \%(18,19)$.

More than half $(58 \%)$ of the isolates from blood were Salmonella species. Isolates from blood showed an excellent sensitivity pattern except ciprofloxacin (7\%) and ampicillin (74\%). In India, Salmonella enterica serovar Typhi and Paratyphi were predominant blood isolates. In S. Typhi and paratyphi, they found nalidixic acid as the most resistant drug with a variable finding of ciprofloxacin (0-81\%). Other drugs showed better sensitivity. Stool constituted $20 \%$ of Salmonella species. Isolates from stool samples here showed a very different scenario with a highest sensitivity (70\%) to ceftriaxone assuming that they might be non typhoidal salmonellosis (NTS). Among NTS from India, susceptibility was observed for ampicillin (0-93\%), co-trimoxazole (4293\%), chloramphenicol (45-100\%), nalidixic acid (23-76\%), ciprofloxacin (9-100\%), ceftriaxone and azithromycin (>90\%) (20-23). Shigella species from stools also have similar sensitivity patterns.

V. Cholera was the most abundant bacteria (48\%) in stool. Two of the major causes of severe diarrhea in low-resource countries, are the bacterial pathogens Vibrio cholerae (O1), which causes epidemic cholera, and enterotoxigenic Escherichia coli (ETEC) (24). We excluded E. coli as there is a lack of capacity to toxigenicity in our laboratory set up. In a systemic surveillance on diarrheal 
medRxiv preprint doi: https://doi.org/10.1101/2021.06.12.21251710; this version posted August 10, 2021. The copyright holder for this preprint (which was not certified by peer review) is the author/funder, who has granted medRxiv a license to display the preprint in perpetuity.

It is made available under a CC-BY 4.0 International license.

patients carried out by icddr, b, it was found that the most prevalent pathogen isolated was Vibrio cholerae $\mathrm{O} 1$ (23\%) followed by ETEC (11\%) (25).

In the present study, Vibrio cholerae was highly susceptible to ciprofloxacin and azithromycin. In India, the antimicrobial susceptibility profile observed were, ampicillin (0-68\%), trimethoprimsulfamethoxazole (0-33\%), chloramphenicol (30-90\%), tetracycline (2-70\%), nalidixic acid (0$66 \%$ ), ciprofloxacin (10-94\%), norfloxacin (14-94\%), ofloxacin (6-90\%) and cefixime (0-95\%) (26-28).

Though Acinetobacter baumannii (ACB) complex comprises only $4 \%$ of total and constituted $26 \%$ of ETA isolates. Susceptibility profile was the worst as expected and showed the most sensitivity to Imipenem by $29 \%$. But studies from India got better susceptibility patterns for imipenem, meropenem, amikacin, tobramycin, netilmicin and colistin compared to our surveillance reports (29-31).

Among the gram positive organisms, Staphylococcus aureus was the most (10\%) prevalent, followed by Enterococcus species and Streptococcus pneumoniae. Staphylococcus aureus isolates were mostly found in respiratory samples followed by wound swab, blood and urine. The overall susceptibility pattern showed the most effective drug was Rifampicin (69\%) and the least was Penicillin-G (11\%). Only 36\% isolates showed sensitivity towards Cefoxitin indicates the high MRSA burden in the region. It still needs to differentiate the healthcare associated and community acquired infections to identify the actual magnitude of the MRSA in these settings. We could not evaluate the source of the infection either hospital origin or community origin. The overall clindamycin susceptibility was higher $(41 \%)$ than the cefoxitin albeit we could not evaluate the clinical significance of these findings due to lack of performing the inducible clindamycin resistance test in our sentinel sites. In India, S. aureus (MSSA) and methicillin-resistant S. aureus 
medRxiv preprint doi: https://doi.org/10.1101/2021.06.12.21251710; this version posted August 10, 2021. The copyright holder for this preprint (which was not certified by peer review) is the author/funder, who has granted medRxiv a license to display the preprint in perpetuity.

It is made available under a CC-BY 4.0 International license .

(MRSA) were reported separately. They found almost $40 \%$ isolates as MRSA. For MRSA, poor susceptibility to gentamicin (28-44\%), erythromycin (9-69\%), clindamycin (35-71\%), cotrimoxazole (27-66\%) and ciprofloxacin (8-21\%) was found (32-34).

Enterococcus isolates were mostly present in the Urine. The most sensitive drug was Nitrofurantoin to be found effective against Enterococcus. Susceptibility patterns in Indian studies were ampicillin (3-35\%), gentamicin (16-89\%), vancomycin (77-100\%) and linezolid (98-100\%). Vancomycin resistance was reported around 20\% (35-37). Only 06 Streptococcus pneumoniae could be isolated from respiratory samples and blood. The fastidious nature of this organism could be underestimated. Very few isolates are not conclusive to evaluate the antibiogram as it was below 30 in number.

\section{Limitations of the study}

As the participatory laboratories were not capable of performing MIC testing, some of the important susceptibility patterns could not be confirmed which required it according to CLSI. Furthermore, the toxigenicity test of stool samples yielding E.coli could not be performed so that one of the important diarrheagenic pathogens had to be excluded from the susceptibility list.

\section{Conclusion}

AMR Surveillance is an essential tool for getting necessary information to develop and monitor therapy guidelines, antibiotic stewardship programmes, public health interventions and infection control policies. It enables early detection of resistant strains of public health importance, and supports the prompt notification and investigation of outbreaks and ultimately guides to policy recommendations. 
medRxiv preprint doi: https://doi.org/10.1101/2021.06.12.21251710; this version posted August 10, 2021. The copyright holder for this preprint (which was not certified by peer review) is the author/funder, who has granted medRxiv a license to display the preprint in perpetuity.

It is made available under a CC-BY 4.0 International license.

Running a standard and reliable surveillance is extremely important as well as challenging especially in low resource countries like Bangladesh. The ongoing AMR surveillance program, with all its limitations and challenges, is unique for its countrywide expansion and coordinated approach of hospital physicians, nurses of medical college hospitals and laboratory personnel of microbiology departments of the medical colleges. The susceptibility pattern of different microorganisms as revealed by the surveillance is indeed alarming. Except Imipenem most of the commonly used antibiotics showed ineffective for most of the bacteria. The Acb complex, most of which organism has been found from ICU patient's specimens, showed less than $50 \%$ sensitivity to all of the used antibiotics. Vibrio cholerae isolated from stool samples showed similar sensitivity to ciprofloxacin (84\%) and azithromycin (83\%) whereas Shigella spp. showed only 53\% sensitivity to azithromycin while ceftriaxone (89\%) showed highest sensitivity. Salmonella spp. from blood showed high sensitivity (70-100\%) to most of the antibiotics except ciprofloxacin (7\%). Staph. aureus is only $36 \%$ sensitive to cefoxitin which indicates the possibility to a very high number of MRSA. Antibiotics like ampicillin, sulfamethoxazole-trimethoprim and tetracycline which was previously used commonly does not seem to be effective to most of the bacteria except salmonella spp. It is high time all relevant stakeholders should come forward to curb the upcoming threat.

\section{Acknowledgement}

The AMR surveillance is funded by the Government of People's Republic of Bangladesh, Centers for Disease Control and Prevention, USA through Global Health Security Agenda and World Health Organizations. The protocol of this surveillance system was developed with the support of American Society for Microbiology. We also acknowledge all the staff of National 
medRxiv preprint doi: https://doi.org/10.1101/2021.06.12.21251710; this version posted August 10, 2021. The copyright holder for this preprint (which was not certified by peer review) is the author/funder, who has granted medRxiv a license to display the preprint in perpetuity.

It is made available under a CC-BY 4.0 International license .

Reference Laboratory of AMR, IEDCR, Bangladesh and staff of the sentinel sites for their intense support for this study.

\section{References}

1. WHO. New report calls for urgent action to avert antimicrobial resistance crisis. 2019.

2. OECD. Stopping antimicrobial resistance would cost just USD 2 per person a year. 2018.

3. Asna S, Haq JA, Rahman MM. Nalidixic acid-resistant Salmonella enterica serovar Typhi with decreased susceptibility to ciprofloxacin caused treatment failure: a report from Bangladesh. Japanese journal of infectious diseases. 2003;56(1):32-3.

4. Shamsuzzaman MM, Biswas TK. Aqua chemicals in shrimp farm: A study from southwest coast of Bangladesh. The Egyptian Journal of Aquatic Research. 2012;38(4):275-85.

5. Afroz S, Habib ZH, Billah SMB, Akhter H, Jahan H, Parveen R. Spectrum and Antibiotic Resistance Pattern of Bacteria Causing Urinary Tract Infections (UTI) in a Tertiary Care Hospital. Journal of Surgical Sciences. 2019;23(1):13-8.

6. Biswas R, Rabbani R, Ahmed HS, Sarker MAS, Zafrin N, Rahman MM. Antibiotic sensitivity pattern of urinary tract infection at a tertiary care hospital. Bangladesh Critical Care Journal. 2014;2(1):21-4.

7. Veeraraghavan B, Walia K. Antimicrobial susceptibility profile \& resistance mechanisms of Global Antimicrobial Resistance Surveillance System (GLASS) priority pathogens from India. The Indian journal of medical research. 2019;149(2):87. 
medRxiv preprint doi: https://doi.org/10.1101/2021.06.12.21251710; this version posted August 10, 2021. The copyright holder for this preprint (which was not certified by peer review) is the author/funder, who has granted medRxiv a license to display the preprint in perpetuity.

It is made available under a CC-BY 4.0 International license.

8. Bora A, Hazarika NK, Shukla SK, Prasad KN, Sarma JB, Ahmed G. Prevalence of blaTEM, blaSHV and blaCTX-M genes in clinical isolates of Escherichia coli and Klebsiella pneumoniae from Northeast India. Indian journal of Pathology and Microbiology. 2014;57(2):249.

9. Ranjan A, Shaik S, Mondal A, Nandanwar N, Hussain A, Semmler T, et al. Molecular epidemiology and genome dynamics of New Delhi metallo- $\beta$-lactamase-producing extraintestinal pathogenic Escherichia coli strains from India. Antimicrobial agents and chemotherapy. 2016;60(11):6795-805.

10. Oguntibeju O, Rau N. Occurrence of Pseudomonas aeruginosa in post-operative wound infection. Pakistan Journal of Medical Sciences. 2004;20:187-92.

11. Rai S, Yadav UN, Pant ND, Yakha JK, Tripathi PP, Poudel A, et al. Bacteriological profile and antimicrobial susceptibility patterns of bacteria isolated from pus/wound swab samples from children attending a tertiary care hospital in Kathmandu, Nepal. International journal of microbiology. 2017;2017.

12. Ahmed NH, Hussain T. Antimicrobial susceptibility patterns of leading bacterial pathogens isolated from laboratory confirmed blood stream infections in a multi-specialty sanatorium. Journal of global infectious diseases. 2014;6(4):141.

13. Rose W, Veeraraghavan B, George B. Bloodstream infections in children with febrile neutropenia: Isolates and their antimicrobial susceptibility profile. Indian journal of cancer. 2015;52(4):495.

14. Frieden T. Antibiotic resistance threats in the United States, 2013. Socinorte, Spain: Centers for Disease Control and Prevention, US Department of Health and Human Services. 2013: 11-28. 
medRxiv preprint doi: https://doi.org/10.1101/2021.06.12.21251710; this version posted August 10, 2021. The copyright holder for this preprint (which was not certified by peer review) is the author/funder, who has granted medRxiv a license to display the preprint in perpetuity.

It is made available under a CC-BY 4.0 International license.

15. Egbe CA, Ndiokwere C, Omoregie R. Microbiology of lower respiratory tract infections in Benin City, Nigeria. The Malaysian journal of medical sciences: MJMS. 2011;18(2):27.

16. Budayanti NS, Suryawan K, Iswari IS, Sukrama DM. The quality of sputum specimens as a predictor of isolated bacteria from patients with lower respiratory tract infections at a tertiary referral hospital, Denpasar, Bali-Indonesia. Frontiers in medicine. 2019;6:64.

17. Joseph NM, Sistla S, Dutta TK, Badhe AS, Rasitha D, Parija SC. Ventilator-associated pneumonia in a tertiary care hospital in India: role of multi-drug resistant pathogens. The Journal of Infection in Developing Countries. 2010;4(04):218-25.

18. Gandra S, Mojica N, Klein EY, Ashok A, Nerurkar V, Kumari M, et al. Trends in antibiotic resistance among major bacterial pathogens isolated from blood cultures tested at a large private laboratory network in India, 2008-2014. International Journal of Infectious Diseases. 2016;50:7582.

19. Veeraraghavan B, Shankar C, Karunasree S, Kumari S, Ravi R, Ralph R. Carbapenem resistant Klebsiella pneumoniae isolated from bloodstream infection: Indian experience. Pathogens and global health. 2017;111(5):240-6.

20. Dahiya S, Sharma P, Kumari B, Pandey S, Malik R, Manral N, et al. Characterisation of antimicrobial resistance in Salmonellae during 2014-2015 from four centres across India: An ICMR antimicrobial resistance surveillance network report. Indian journal of medical microbiology. 2017;35(1):61-8.

21. Dutta S, Das S, Mitra U, Jain P, Roy I, Ganguly SS, et al. Antimicrobial resistance, virulence profiles and molecular subtypes of Salmonella enterica serovars Typhi and Paratyphi A blood isolates from Kolkata, India during 2009-2013. PloS one. 2014;9(8):e101347. 
medRxiv preprint doi: https://doi.org/10.1101/2021.06.12.21251710; this version posted August 10, 2021. The copyright holder for this preprint (which was not certified by peer review) is the author/funder, who has granted medRxiv a license to display the preprint in perpetuity.

It is made available under a CC-BY 4.0 International license.

22. Rudresh SM, Nagarathnamma T. Antibiotic susceptibility pattern of Salmonella enterica serovar typhi and Salmonella enterica serovar paratyphi A with special reference to quinolone resistance. Drug Development \& Therapeutics. 2015;6(2).

23. Taneja N, Appannanavar SB, Kumar A, Varma G, Kumar Y, Mohan B, et al. Serotype profile and molecular characterization of antimicrobial resistance in non-typhoidal Salmonella isolated from gastroenteritis cases over nine years. Journal of medical microbiology. 2014;63(1):66-73.

24. Begum YA, Rydberg HA, Thorell K, Kwak Y-K, Sun L, Joffré E, et al. In situ analyses directly in diarrheal stool reveal large variations in bacterial load and active toxin expression of enterotoxigenic Escherichia coli and Vibrio cholerae. Msphere. 2018;3(1):e00517-17.

25. Chowdhury F, Rahman MA, Begum YA, Khan AI, Faruque AS, Saha NC, et al. Impact of rapid urbanization on the rates of infection by Vibrio cholerae $\mathrm{O} 1$ and enterotoxigenic Escherichia coli in Dhaka, Bangladesh. PLoS neglected tropical diseases. 2011;5(4):e999.

26. Aggarwal P, Uppal B, Ghosh R, Jha AK, Konar D. Surveillance of changing antimicrobial resistance pattern in Shigella in North India. Int J. 2014;2:166-71.

27. Anandan S, Sethuvel DPM, Gajendiren R, Verghese VP, Walia K, Veeraraghavan B. Molecular characterization of antimicrobial resistance in clinical Shigella isolates during 2014 and 2015: trends in South India. Germs. 2017;7(3):115.

28. Das A, Natarajan M, Mandal J. The emergence of quinolone resistant Shigella sonnei, Pondicherry, India. PLoS One. 2016;11(8):e0160290. 
medRxiv preprint doi: https://doi.org/10.1101/2021.06.12.21251710; this version posted August 10, 2021. The copyright holder for this preprint (which was not certified by peer review) is the author/funder, who has granted medRxiv a license to display the preprint in perpetuity.

It is made available under a CC-BY 4.0 International license .

29. Pragasam A, Vijayakumar S, Bakthavatchalam Y, Kapil A, Das B, Ray P, et al. Molecular characterisation of antimicrobial resistance in Pseudomonas aeruginosa and Acinetobacter baumannii during 2014 and 2015 collected across India. Indian journal of medical microbiology. 2017;34(4):433-41.

30. Shrivastava G, Bhatambare GS, Bajpai T, Patel KB. Sensitivity profile of multidrug resistant Acinetobacter Spp. isolated at ICUs of tertiary care hospital. International Journal of Health System and Disaster Management. 2013;1(4):200.

31. Tripathi PC, Gajbhiye SR, Agrawal GN. Clinical and antimicrobial profile of Acinetobacter spp.: An emerging nosocomial superbug. Advanced biomedical research. 2014;3.

32. India SJ, Ray P, Manchanda V, Bajaj J, Chitnis D, Gautam V, et al. Methicillin resistant Staphylococcus aureus (MRSA) in India: prevalence \& susceptibility pattern. The Indian journal of medical research. 2013;137(2):363.

33. Moolchandani K, Sastry AS, Deepashree R, Sistla S, Harish B, Mandal J. Antimicrobial resistance surveillance among intensive care units of a tertiary care hospital in Southern India. Journal of clinical and diagnostic research: JCDR. 2017;11(2):DC01.

34. Rajkumar S, Sistla S, Manoharan M, Sugumar M, Nagasundaram N, Parija SC, et al. Prevalence and genetic mechanisms of antimicrobial resistance in Staphylococcus species: A multicentre report of the Indian council of medical research antimicrobial resistance surveillance network. Indian journal of medical microbiology. 2017;35(1):53-60.

35. Praharaj I, Sujatha S, Parija SC. Phenotypic \& genotypic characterization of vancomycin resistant Enterococcus isolates from clinical specimens. The Indian journal of medical research. 2013;138(4):549. 
medRxiv preprint doi: https://doi.org/10.1101/2021.06.12.21251710; this version posted August 10, 2021. The copyright holder for this preprint (which was not certified by peer review) is the author/funder, who has granted medRxiv a license to display the preprint in perpetuity.

It is made available under a CC-BY 4.0 International license.

36. Sachan S, Rawat V, Umesh MK, Kaur T, Chaturvedi P. Susceptibility pattern of enterococci at tertiary care hospital. Journal of global infectious diseases. 2017;9(2):73.

37. Singh AK, Venkatesh V, Singh RP, Singh M. Bacterial and antimicrobial resistance profile of bloodstream infections: a hospital-based study. CHRISMED Journal of Health and Research. 2014;1(3):140. 